\title{
NTRK2 expression levels are reduced in laser captured pyramidal neurons from the anterior cingulate cortex in males with autism spectrum disorder
}

Michelle J Chandley ${ }^{1}$, Jessica D Crawford ${ }^{2}$, Attila Szebeni ${ }^{2}$, Katalin Szebeni ${ }^{2}$ and Gregory A Ordway ${ }^{2 *}$

\begin{abstract}
Background: The anterior cingulate cortex (ACC) is a brain area involved in modulating behavior associated with social interaction, disruption of which is a core feature of autism spectrum disorder (ASD). Functional brain imaging studies demonstrate abnormalities of the ACC in ASD as compared to typically developing control patients. However, little is known regarding the cellular basis of these functional deficits in ASD. Pyramidal neurons in the ACC are excitatory glutamatergic neurons and key cellular mediators of the neural output of the ACC. This study was designed to investigate the potential role of ACC pyramidal neurons in ASD brain pathology.
\end{abstract}

Methods: Postmortem ACC tissue from carefully matched ASD and typically developing control donors was obtained from two national brain collections. Pyramidal neurons and surrounding astrocytes were separately collected from layer III of the ACC by laser capture microdissection. Isolated RNA was subjected to reverse transcription and endpoint PCR to determine gene expression levels for 16 synaptic genes relevant to glutamatergic neurotransmission. Cells were also collected from the prefrontal cortex (Brodmann area 10) to examine those genes demonstrating differences in expression in the ACC comparing typically developing and ASD donors.

Results: The level of NTRK2 expression was robustly and significantly lower in pyramidal neurons from ASD donors as compared to typically developing donors. Levels of expression of GRIN1, GRM8, SLCIA1, and GRIP1 were modestly lower in pyramidal neurons from ASD donors, but statistical significance for these latter genes did not survive correction for multiple comparisons. No significant expression differences of any genes were found in astrocytes laser captured from the same neocortical area. In addition, expression levels of NTRK2 and other synaptic genes were normal in pyramidal neurons laser captured from the prefrontal cortex.

Conclusions: These studies demonstrate a unique pathology of neocortical pyramidal neurons of the ACC in ASD. NTRK2 encodes the tropomyosin receptor kinase B (TrkB), transmission through which neurotrophic factors modify differentiation, plasticity, and synaptic transmission. Reduced pyramidal neuron NTRK2 expression in the ACC could thereby contribute to abnormal neuronal activity and disrupt social behavior mediated by this brain region.

Keywords: Pyramidal neurons, Astrocytes, Cingulate, Autism, Glutamate receptors

\footnotetext{
* Correspondence: ordway@etsu.edu

${ }^{2}$ Department of Biomedical Sciences, James H. Quillen College of Medicine, East Tennessee State University, P.O. Box 70582, Johnson City, TN 37614, USA Full list of author information is available at the end of the article
} 


\section{Background}

Autism spectrum disorder (ASD) is a neurodevelopmental disorder that includes repetitive behaviors and impairments in social communication and interaction [1]. In recent years, ASD and disorders associated with autism behaviors have largely been attributed to genetic etiology [2]. However, it is likely that the heterogeneity of ASD results from a complex interplay of inherited genetics and developmental influences that result in abnormal intercellular communication in the brain. It is anticipated that areas of the brain that modulate the behaviors that are disrupted in ASD are particularly vulnerable to the pathobiological processes of ASD and likely include disrupted connectivity between discrete areas of the brain that modulate behaviors that are abnormal from infancy.

The anterior cingulate cortex (ACC) is the cortical gyrus surrounding the corpus callosum in the human brain. Anatomically, the ACC shares a complex relationship with several other brain regions including the amygdala, hypothalamus, parietal, and other regions of the prefrontal cortex (as reviewed by Bush et al., 2000 [3]). The ACC has consistently displayed abnormalities in ASD as revealed through imaging and neuroanatomical studies. Functional MRI studies have demonstrated differences when comparing typically developed controls with ASD patients, including reduced activation of the anterior cingulate cortex (ACC) during true/false judgments [4], novelty detection [5], response inhibition [6,7], attention tasks [8], and increased activation of the ACC during social target detection and nonsocial rewarding [9]. Other studies using SPECT [10], EEG [11-14], PET [15-18], and fractional anisotropy $[19,20]$ have also demonstrated abnormalities in the cingulate cortex in ASD.

The few postmortem studies that have focused on the ACC have identified molecular and anatomical differences between ASD and typically developing control brain tissue. Stereology studies using the neocortex of the ACC indicated that neurons were smaller and demonstrated increased cell packing density [21]. Reductions in both mRNA and protein levels of the axonal guidance proteins plexinA4 and roundabout 2 were identified in tissue homogenates from the ACC from ASD donors [22], as well as alterations in serotonin- [23] and GABA- [24,25] related genes. Interestingly, increased gene expression in the transcriptional control factor, Sp1, was found in postmortem ACC from ASD donors [26], which could have widespread implications and contribute to the complexity of ASD. Collectively, these studies demonstrate that pathology in the ACC exists in autism. However, little is known regarding the cellular basis of ACC pathology.

Pyramidal neurons in layer III of the neocortex are key cellular mediators of the neural output of the ACC. These pyramidal cells are excitatory glutamatergic neurons that have a complex synaptic relationship with many other cell types in other neocortical layers of the ACC, including inhibitory neurons, glia, and long-range projecting glutamate neurons of layer $\mathrm{V}$. The current study was undertaken to investigate the molecular pathology of pyramidal cells in layer III of the ACC from ASD donors. The levels of expression of several glutamaterelated genes were measured specifically in pyramidal neurons captured by laser microdissection from layer III of the ACC from postmortem brain tissue from ASD donor and typically developing control subject brain tissue. The glutamate-related genes chosen for study were those associated with ASD identified in gene association, laboratory animal, and/or postmortem pathology studies [27-32] and/or because of their strong association with glutamatergic neurotransmission. Additionally, two neurotrophic factor genes (brain-derived neurotrophic factor (BDNF) and NTRK2) were studied because of the link between glutamatergic transmission and BDNF signaling [33] and the association of BDNF and NTRK2 pathology in ASD [34]. The findings of this study demonstrate that ACC in ASD is associated with abnormal levels of expression of several genes related to glutamate neurotransmission, with the most striking finding being a robust reduction of NTRK2 gene expression.

\section{Methods}

\section{Brain tissue}

Brain tissues from ASD and typically developing control donors were provided by the National Institutes for Child Health and Development (NICHD) Brain and Tissue Bank (Baltimore, MD) and the Autism Tissue Program (Belmont, MA). These brain banks were responsible for obtaining subject consent and the unidentifiable coding of subject information. This study was reviewed and approved for exemption by the Institutional Review Board of East Tennessee State University under the Department of Heath and Human Services exemption 45 CFR 46.101(b) relating to the use of publicly available unidentifiable pathology specimens. In total, brain tissue from 12 typically developing control donors and 12 ASD donors were used for the different experiments (see Table 1). Comorbidities and causes of death were not included in the table in order to protect donor identities. Typically developing control donors died by drowning (3 donors), heart condition (3 donors), trauma (3 donors), asphyxia (1 donor), pneumonia (1 donor), and unknown cause (1 donor). It should be mentioned that one control donor was diagnosed with depressive disorders and died by suicide. The ASD donors died by trauma (3 donors), asphyxia (3 donors), acute respiratory distress syndrome (1 donor), cardiopulmonary arrest (1 donor), cancer (1 donor), diabetic ketoacidosis (1 donor), bowel obstruction (1 donor), and cardiac arrhythmia (1 donor). One ASD donor could not be medically confirmed as 
Table 1 Subject demographic information

\begin{tabular}{|c|c|c|c|c|c|c|c|c|}
\hline Pair & ID & Age & Gender & $\mathrm{RIN}^{\mathrm{a}}$ & PMI (hours) ${ }^{b}$ & Toxicology & Assays & Tissue \\
\hline \multicolumn{9}{|c|}{ Control donors } \\
\hline 1 & AN14757 & 24 & M & 7.8 & 21.33 & No drugs reported & $L C M^{c}, q^{\prime} C R^{d}$ & $\mathrm{BA} 24, \mathrm{BA} 10$ \\
\hline 2 & AN07176 & 21 & M & 8 & 29.91 & No drugs reported & $\mathrm{LCM}, \mathrm{qPCR}$ & $\mathrm{BA} 24, \mathrm{BA} 10$ \\
\hline 3 & AN07444 & 17 & M & 7.5 & 30.75 & Sertraline & LCM, qPCR & BA24 \\
\hline 4 & 5408 & 6 & M & 7 & 16 & No drugs reported & $\mathrm{LCM}, \mathrm{qPCR}$ & $\mathrm{BA} 24, \mathrm{BA} 10$ \\
\hline 5 & 4848 & 16 & M & 7.6 & 15 & No drugs reported & LCM, qPCR & $\mathrm{BA} 24, \mathrm{BA} 10$ \\
\hline 6 & 5342 & 22 & M & 8.1 & 14 & No drugs reported & $\mathrm{LCM}, \mathrm{qPCR}$ & $\mathrm{BA} 24, \mathrm{BA} 10$ \\
\hline 7 & 5079 & 33 & M & 7.3 & 16 & Ethanol & LCM, qPCR & $\mathrm{BA} 24, \mathrm{BA} 10$ \\
\hline 8 & M3231M & 37 & M & 7.4 & 24 & No drugs reported & $L C M, q P C R$ & $\mathrm{BA} 24, \mathrm{BA} 10$ \\
\hline 9 & 4590 & 20 & M & 7.6 & 19 & No drugs reported & LCM, $\mathrm{qPCR}$ & BA10 \\
\hline \multicolumn{2}{|c|}{ Mean \pm SEM (BA24 donors) } & \multicolumn{2}{|l|}{$22.0 \pm 3.4$} & $7.59 \pm 0.13$ & $20.9 \pm 2.4$ & & & \\
\hline \multicolumn{2}{|c|}{ Mean \pm SEM (BA10 donors) } & \multicolumn{2}{|l|}{$22.4 \pm 3.4$} & $7.60 \pm 0.13$ & $19.4 \pm 1.9$ & & & \\
\hline \multicolumn{9}{|c|}{ ASD donors } \\
\hline 1 & AN04166 & 24 & M & 8.1 & 18.51 & No drugs reported & LCM, qPCR & $\mathrm{BA} 24, \mathrm{BA} 10$ \\
\hline 2 & AN03935 & 20 & M & 8.6 & 28 & No drugs reported & LCM, qPCR & $\mathrm{BA} 24, \mathrm{BA} 10$ \\
\hline 3 & AN02987 & 15 & M & 6.5 & 30.83 & No drugs reported & LCM, qPCR & BA24 \\
\hline 4 & 5144 & 7 & M & 8.0 & 3 & No drugs reported & LCM, qPCR & $\mathrm{BA} 24, \mathrm{BA} 10$ \\
\hline 5 & 5302 & 16 & M & 6.6 & 20 & $\begin{array}{l}\text { Risperidone, fluvoxamine, } \\
\text { clonidine, insulin }\end{array}$ & LCM, qPCR & $\mathrm{BA} 24, \mathrm{BA} 10$ \\
\hline 6 & 5176 & 22 & M & 7.1 & 18 & Risperidone & LCM, qPCR & $\mathrm{BA} 24, \mathrm{BA} 10$ \\
\hline 7 & 5297 & 33 & M & 7.1 & 50 & $\begin{array}{l}\text { Quetiapine, fluoxetine, } \\
\text { valproate, ziprasidone }\end{array}$ & LCM, qPCR & $\mathrm{BA} 24, \mathrm{BA} 10$ \\
\hline 8 & 5027 & 37 & M & 7.7 & 26 & Risperidone, fluvoxamine & LCM, qPCR & $\mathrm{BA} 24, \mathrm{BA} 10$ \\
\hline 9 & 4999 & 20 & M & 7.0 & 14 & No drugs reported & LCM, qPCR & BA10 \\
\hline \multicolumn{2}{|c|}{ Mean \pm SEM (BA24 donors) } & $21.6 \pm 3.4$ & & $7.46 \pm 0.27$ & $24.3 \pm 4.8$ & & & \\
\hline \multicolumn{2}{|c|}{ Mean \pm SEM (BA10 donors) } & $22.2 \pm 3.3$ & & $7.53 \pm 0.24$ & $23.4 \pm 5.4$ & & & \\
\hline \multicolumn{2}{|c|}{ P value (BA 24) } & 0.94 & & 0.68 & 0.53 & & & \\
\hline \multicolumn{2}{|c|}{ P value $e^{e}$ (BA10) } & 0.98 & & 0.79 & 0.60 & & & \\
\hline
\end{tabular}

${ }^{a}$ RNA integrity number (index of RNA quality); ${ }^{b}$ postmortem interval; ${ }^{c}$ endpoint PCR analysis of reversed transcribed RNA isolated from cells collected by laser capture microdissection; ${ }^{\mathrm{d}}$ quantitative polymerase chain reaction of reverse transcribed RNA isolated from punch-dissected tissues; ${ }^{\mathrm{e}}$ results of a two-tailed independent $t$-test comparing control and ASD groups. BA24, Brodmann area 24; BA10, Brodmann area 10; LCM, laser capture microdissection.

ASD after death, and one ASD donor had a single seizure episode but did not have a medical diagnosis of seizure disorder. The samples were closely matched by gender and age. For each control and ASD pair, the donor tissue came from the same brain bank and was not anatomically characterized by subregions of Brodmann area 24 (BA24) or Brodmann area 10 (BA10). Additionally, we analyzed RNA integrity values (RIN, index of RNA quality) in matched pairs of donors prior to experimentation to be sure these were closely matched for the paired analyses [35].

\section{Tissue preparation}

Blocks of tissue containing ACC, specifically BA24, and prefrontal cortex BA10 were received and stored at $-80^{\circ} \mathrm{C}$. All brain tissues were obtained from the left hemisphere except AN03935. Tissue homogenates used for quantitative PCR were obtained using a $3.5-\mathrm{mm}$ trephine to punch-dissect a $50-\mu \mathrm{m}$-thick section of gray matter from the anterior cingulate that contained all six neocortical layers. A cryostat microtome was used to section tissue blocks for laser capture microdissection (LCM). Tissue from the cortex was sectioned at $-20^{\circ} \mathrm{C}$ and $10-\mu \mathrm{m}$-thick sections were mounted slides that were immediately placed in a chilled microslide box on ice. Between each tissue block, all physical elements of cryosectioning were thoroughly cleaned with 100\% ethanol to avoid any cross contamination. Sectioned tissue was desiccated at room temperature for $5 \mathrm{~min}$ and stored at $-80^{\circ} \mathrm{C}$ until use. Tissues from the same control/ASD donor pair were sectioned and processed on the same day to ensure that storage time after sectioning was identical. 


\section{Laser capture microdissection}

Neurons were visualized by staining frozen tissue sections with the Histogene staining kit (Life Technologies; Grand Island, NY, USA) according to the manufacturer's instructions (Figure 1C). In short, the protocol used a cresyl violet stain on an ethanol-fixed slide followed by a series of dehydration steps. Stained slides were placed in a vacuum chamber until ready for LCM. Astrocytes were identified using a modified glial fibrillary acidic protein (GFAP) rapid immunohistochemistry protocol as previously described (Figure 1E) [36,37]. Briefly, tissue-mounted slides were fixed in acetone (5 min), blocked in horse serum (10 min), incubated with GFAP antibody (10 min; ThermoFisher; Pittsburgh, PA, USA), anti-mouse antibody (5 min; Vectastain; Burlingame, CA, USA), and avidinbiotinylated horseradish peroxidase (5 min; Vectastain; Burlingame, CA, USA). GFAP immunoreactivity was visualized by exposure to diaminobenzidine (DAB) (Sigma; St. Louis, MO, USA) with ammonium nickel sulfate (ANS) (Sigma; St. Louis, MO, USA) for $5 \mathrm{~min}$, then exposed to DAB/ANS with $0.1 \% \mathrm{H}_{2} \mathrm{O}_{2}$ for an additional 5 min. Afterwards, the slide was ethanol dehydrated then placed in xylene for $5 \mathrm{~min}$. The slide was kept in a vacuum chamber for $10 \mathrm{~min}$ prior to LCM.

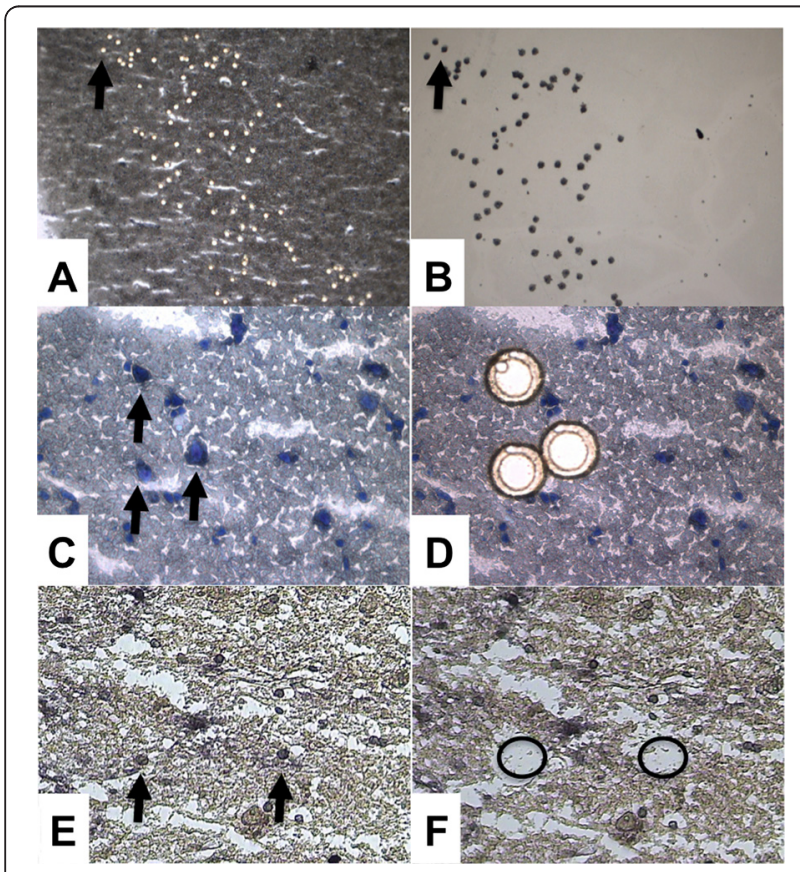

Figure 1 Laser capture microdissection of pyramidal neurons and astrocytes. Shown in (A) is the absence of laser captured nisslstained pyramidal neurons from cortical layer 3 in BA24 gray matter tissue following capture (20x magnification). (B) shows those cells adhered to the polymer cap (20x magnification). (C) and (D) illustrate the before (C) and after (D) capture images for nissl-stained BA24 pyramidal neurons (40X magnification). (E) and (F) show the same laser capture process for GFAP immunostained astrocytes in BA24 gray matter (60× magnification).
LCM was performed using an ArcturusXT (Life Technologies; Grand Island, NY, USA) instrument. Neurons were extracted onto CapSure macrocaps (Life Technologies; Grand Island, NY, USA) using a $25-\mu \mathrm{m}$ spot size that is first placed onto the cap by infrared (IR) laser spotting and then cut by an ultraviolet (UV) laser. Neurons were morphologically identified in layer III of the neocortex at 40× magnification. Astrocytes were captured using an Arcturus Veritas (Life Technologies; Grand Island, NY, USA) instrument. Astrocytes were morphologically identified at $60 \times$ magnification and placed onto caps by IR laser using a $10-\mu \mathrm{m}$ spot size. Cells were removed from cap using lysis buffer incubation at $42^{\circ} \mathrm{C}$.

\section{RNA preparation and reverse transcriptase}

Total RNA was extracted from tissue homogenates using the Maxwell simplyRNA LEV kit (Promega; Madison, WI, USA) and from laser captured cells using the RNAqueous Micro kit with DNase treatment (Life Technologies; Grand Island, NY, USA). RNA quality was assessed by measuring RIN values obtained using the Agilent 2100 Bioanalyzer (Agilent Technologies; Santa Clara, CA, USA). RNA samples were reverse transcribed into cDNA using the Superscript III kit (Life Technologies; Grand Island, NY, USA) that contained oligodT and random hexamer primers.

\section{Quantitative PCR}

Gene-specific primers were either designed using Mfold web server software [38] and primer quest design software (IDT; Coralville, IA, USA) to generate approximately 100 base pair amplicons to span exon junctions or were purchased from a vendor (Qiagen; Valencia, CA, USA). Gene primer sequences are shown in Additional file 1. To quantify transcripts, endpoint PCR was used for RNA isolated from laser captured cells and real-time quantitative PCR (qPCR) was used for RNA isolated from tissue homogenates as previously described [36,39]. For endpoint PCR reactions (BDNF, GRIN2D, GRIN2B, and GRM8) that were initially problematic using 5Prime Hot Master Mix taq polymerase (5Prime; Gaithersburg, MD, USA), a modified polymerase from Qiagen (Valencia, CA, USA) was employed using the same reaction parameters.

\section{Statistical analysis}

Calculations for qPCR data involved converting cycle threshold (CT) values to fold-change between control and autism donors using the $2^{-\Delta \Delta C T}$ method by Livak and Schmittgen [40]. Endpoint PCR data was computed as relative values generated from the ratios of amounts of target gene expression to the average of two reference gene expressions. Afterwards, both data from qPCR and endpoint PCR were analyzed by the paired Student $t$-test. Statistical results are reported before and after Holm's 
Bonferroni correction $[41,42]$ for the number of comparisons as noted in the results below. Pearson's correlation was used to determine possible effects of postmortem variables (age, RIN, and postmortem interval time (PMI)) on the expression of each gene. Given the number of correlation tests, a $P<0.01$ was chosen a priori to indicate statistical significance in order to reduce type I errors. Multivariate analysis of variance (MANOVA) for unpaired data was performed using IBM SPSS Statistics (version 21.0.0.0, IBM, New York, NY, USA) and graphed using Prism (version 5.0b, GraphPad Software, La Jolla, CA, USA). All other analyses were performed and graphed using Prism.

\section{Results}

\section{Glutamate-related gene expression}

The levels of expression of seven ionotropic glutamate receptor subunit genes (GRIN1, GRIN2A, GRIN2B, GRIN2C, GRIN2D, GRIK2, GRIA1) and two metabotropic glutamate receptor genes (GRM5, GRM8) were measured in BA24 pyramidal neurons and surrounding astrocytes from ASD and age-matched control donors. GRM8 expression was not detectable in astrocytes. Levels of GRM8 expression were modestly lower in pyramidal neurons from ASD donors compared to matched control donors $(t=2.89 ; P=0.034)$, but statistical significance was lost when the $P$ value was corrected for the number of matched pair comparisons of gene expressions in neurons (Table 2). Likewise, GRIN1 expression levels trended towards being lower in ASD as compared to control donors $(P=0.053)$, but this difference was lost upon correction for the number of comparisons (Table 2). No other differences in the levels of expression of any glutamate receptor gene were observed in either pyramidal neurons or astrocytes comparing ASD to control donors (Figure 2).

Additional genes associated with glutamatergic neurotransmission were interrogated in BA24 pyramidal neurons and astrocytes, including glutamate transporter genes, SLC1A1, SLC1A2, SLC1A3, SLC17A7, and the glutamate receptor interacting gene, GRIP1. Of these genes, the expression of astrocyte-associated transporter genes SLC1A2 and SLC1A3 was not measured in neurons and the neuronal glutamate transporter gene $S L C 1 A 1$ was not measured in astrocytes. Levels of $\operatorname{SLC1A1}(t=2.88$; $P=0.024)$ and GRIP1 $(t=3.84 ; P=0.006)$ gene expression were lower in pyramidal neurons captured from ASD donors as compared to matched control donors (Figure 3). However, statistical significance was lost when $P$ values were corrected for the number of matched pair comparisons (Table 2). No significant differences in the levels of expression of any of the glutamate-related genes, SLC1A2, SLC17A7, and GRIP1,
Table 2 Results of Holm-Bonferroni sequential correction of multiple paired Student's $\boldsymbol{t}$-tests of gene expression data from BA24 neurons

\begin{tabular}{|c|c|c|c|}
\hline Gene (protein) & $\begin{array}{l}P \text { value from } \\
\text { paired } t \text {-test }\end{array}$ & $P^{\prime}$ from correction ${ }^{a}$ & Significance? \\
\hline RNA18S1/GAPDH & 0.3774 & 1.000 & No \\
\hline GRIN1 (NR1) & 0.0533 & 0.583 & No \\
\hline GRIN2A (NR2A) & 0.9051 & 1.000 & $\mathrm{No}$ \\
\hline GRIN2B (NR2B) & 0.1160 & 1.000 & No \\
\hline GRIN2C (NR2C) & 0.9924 & 1.000 & No \\
\hline GRIN2D (NR2D) & 0.1146 & 1.000 & No \\
\hline GRIA1 (GluR-1) & 0.2424 & 1.000 & No \\
\hline GRIK2 (GRIK2, GluK2) & 0.3793 & 1.000 & No \\
\hline GRM5 (mGluR5) & 0.1061 & 1.000 & No \\
\hline GRM8 (mGluR8) & 0.0340 & 0.408 & No \\
\hline SLC1A1 (EAAT3) & 0.0235 & 0.312 & No \\
\hline SLC17A7 (VGlut1) & 0.5034 & 1.000 & No \\
\hline GRIP1 (GRIP1) & 0.0064 & 0.090 & No \\
\hline$B D N F(B D N F)$ & 0.4846 & 1.000 & No \\
\hline NTRK2 (NTRK2,TrkB) & 0.0006 & 0.009 & Yes \\
\hline
\end{tabular}

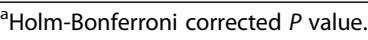

were observed in astrocytes comparing ASD to matched control donors (Figure 3).

\section{BDNF/NTRK2}

Levels of expression for the neurotrophic factor gene $B D N F$ and its receptor gene NTRK2 were measured in pyramidal neurons and astrocytes from BA24 (Figure 4). $B D N F$ expression in both neurons and astrocytes was similar comparing ASD to matched control donors. However, NTRK2 expression levels were robustly lower in pyramidal neurons $(t=5.87 ; P=0.0006)$, but not astrocytes, from ASD donors as compared to control donors. The difference in NTRK2 expression levels between ASD and control donors remained statistically significant after correction for the number of matched comparisons (Table 2).

\section{Expression of selected genes in BA10}

The expression levels of five genes were studied in BA10 pyramidal neurons from ASD and control donors. Chosen for study were those genes analyzed in BA24 pyramidal neurons that demonstrated either no difference (GRM5), marginal or modest differences (GRIN1, SLC1A1), or highly significant differences (NTRK2) comparing ASD to control donors as noted above. In this set of experiments, BA10 tissue from one age-matched pairs of donors used in the BA24 studies above was not available; BA10 tissue from one different age-matched pair of donors was substituted as noted in Table 1. The expression levels of each of these five genes in laser captured 

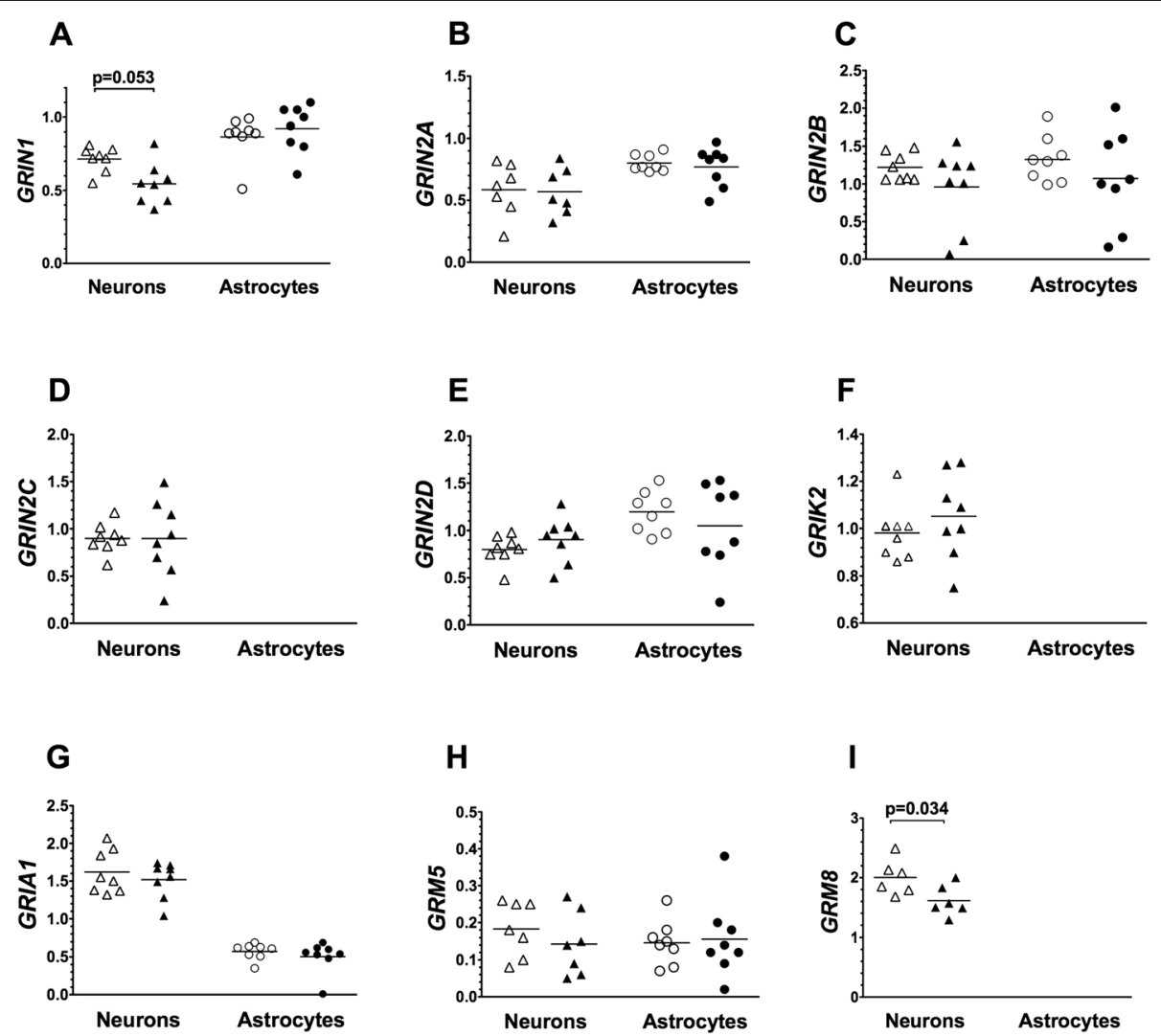

Figure $\mathbf{2}$ Levels of expression of ionotropic glutamate receptor subunits and metabotropic glutamate receptors (A-I). Expression of glutamate receptors and receptor subunits was measured in laser captured BA24 pyramidal neurons and separately in surrounding astrocytes of typically developing control donors (open symbols) and ASD donors (closed symbols). Gene expression levels are normalized to the averaged levels of expression of references genes (GAPDH and RNA18S1). Mean values are noted by horizontal lines, and statistical significance (uncorrected for the number of comparisons) is noted above the data points. See Table 2 for statistical results following correction for the number of comparisons.

pyramidal neurons from BA10 were similar in ASD and matched controls (Figure 5; GRIN1 $t=0.74, P=0.49$; GRM5 $t=0.90, P=0.40$; SLC1A1 $t=0.26, P=0.80$; NTRK2 $t=0.03, P=0.97)$.

Demographic variables, reference genes, and tissue factors There were no statistical differences between ASD and matched control donors of BA24 tissue regarding age, RIN or PMI values (Table 1). Likewise, there were no differences in these variables comparing the groups of donors of BA10 tissue (Table 1). There were no significant correlations between age or PMI and any of the 15 gene expressions measured in BA24 pyramidal neurons (Additional file 2). There was a significant correlation between levels of GRIN2C expression and RIN values, but there were no other correlations between RIN and the levels of any mRNA that was studied in BA24 pyramidal neurons. In BA24 astrocytes, age correlated significantly with NTRK2 gene expression levels $(P=0.002)$ but not with levels of any other gene (Additional file 3 ). RIN did not significantly correlate with expression levels of any gene in BA24 astrocytes, while PMI correlated with only SLC17A7 gene expression in BA24 astrocytes (Additional file 3). In BA10 pyramidal neurons, there were no significant correlations between age, RIN, or PMI and the levels of expression of any of the target or reference genes (Additional file 4).

Reference genes were carefully chosen as those that were stable in their expression levels across the two groups of donors. The ratios of levels of expression of RNA18S1 and GAPDH were not significantly different in laser-captured BA24 neurons $(t=1.00, P=0.35)$, BA24 astrocytes $(t=0.93, P=0.38)$, or BA10 neurons $(t=1.82$, $P=0.11)$ comparing ASD and matched control donors (see Additional file 5).

It should be noted that we wished to screen multiple brain regions for these gene expression changes to determine the extent to which they occurred in the brain. Because LCM is time-intensive and expensive, we attempted to measure SLC1A1, GRIP1, and NTRK2 expression in RNA isolated from homogenates of ACC. However, we were unable to detect any differences in expression of these genes in RNA isolates from homogenates of ACC, using tissues collected from seven of the eight ASD - control 


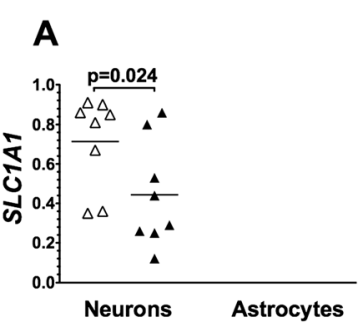

\section{B}
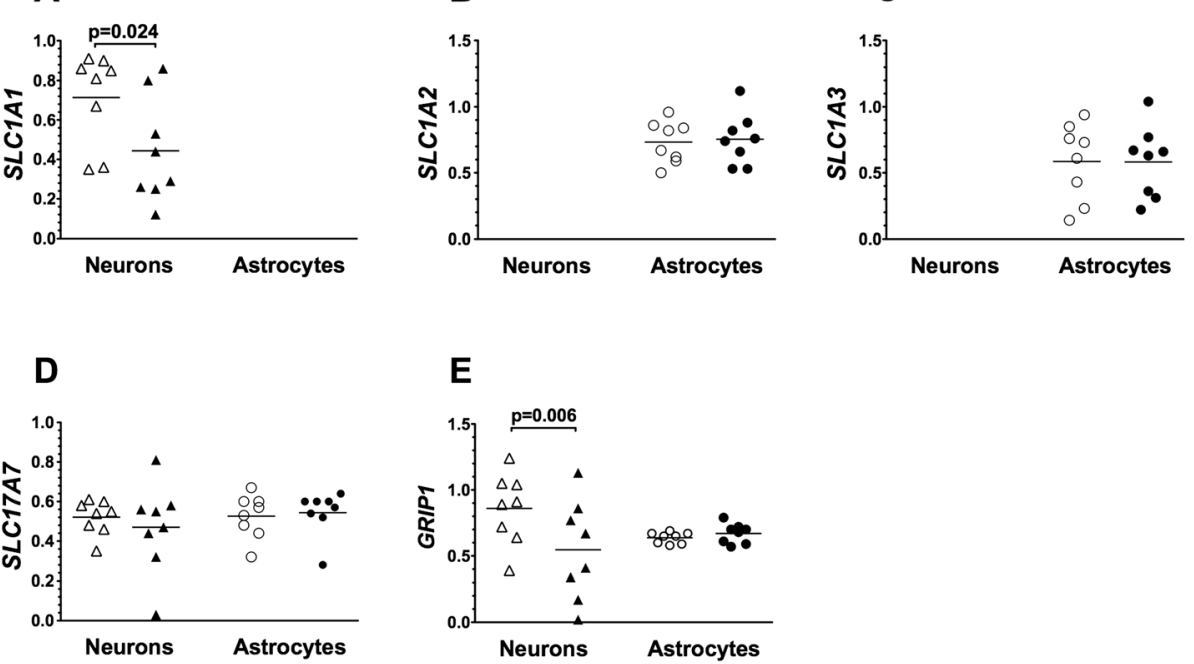

\section{E}

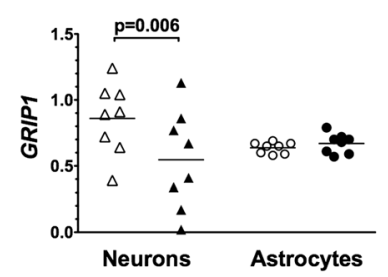

Figure 3 Levels of expression of glutamate transporter genes and a glutamate receptor-interacting gene (A-E). Gene expression was measured in laser captured BA24 pyramidal neurons and separately in surrounding astrocytes of typically developing control donors (open symbols) and ASD donors (closed symbols). Gene expression levels are normalized to the averaged levels of expression of references genes (GAPDH and RNA18S1). Mean values are noted by horizontal lines, and statistical significance (uncorrected for the number of comparisons) is noted above the data points. See Table 2 for statistical results following correction for the number of comparisons.

donor pairs that were used for the LCM study above (Figure 6). No significant difference was found for the ratios of expression of the reference genes GAPDH and TATA from the homogenate punch samples when comparing ASD and control donors (Additional file 6; $t=0.19$, $P=0.86$ ).

Four of the ASD donors had been prescribed psychotherapeutic medications, with three of the four receiving selective serotonin retake inhibitors (SSRI) antidepressants and four of the four receiving antipsychotic drugs (see Table 1). Considering that effects of psychotherapeutic drugs might contribute to observed differences in gene expression in BA24 neurons as shown above, a comparison was made of antidepressant/antipsychoticexposed ASD donors to ASD donors not exposed to these drugs. Albeit the sample sizes of these two groups are small and that these two subgroups were not matched for age and other demographic variables, there were no significant difference in levels of GRM8 (unpaired $t$-test; $t=0.17$, $P=0.87), \operatorname{GRIP1}(0.01, P=0.99), \operatorname{SLC1A1}(t=0.28, P=$ $0.79)$, and NTRK2 $(t=0.59, P=0.57)$ comparing antidepressant/antipsychotic-exposed ASD donors to ASD donors without these drugs.

\section{Statistical considerations}

Data from the matched control and ASD cases were analyzed using a paired $t$-test. Paired analyses were chosen because ASD and control donors were matched prior to the initiation of the analyses of gene expression levels based on several factors including, gender, age, and RNA

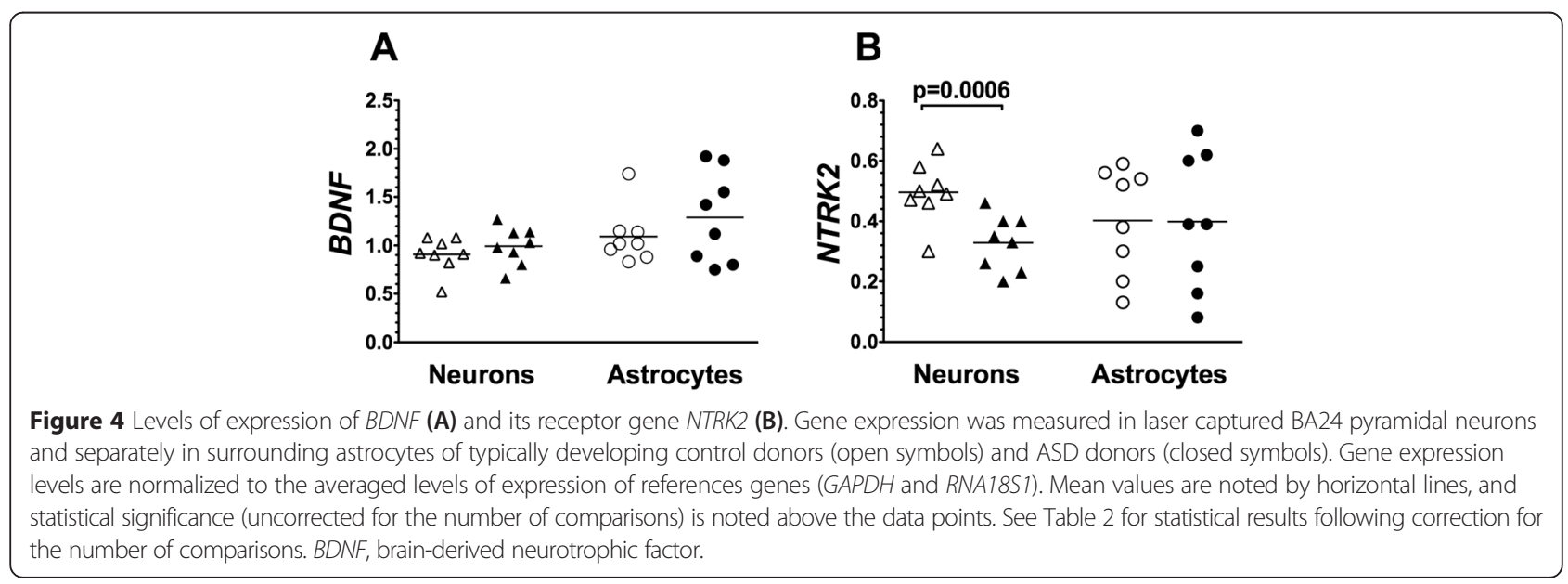




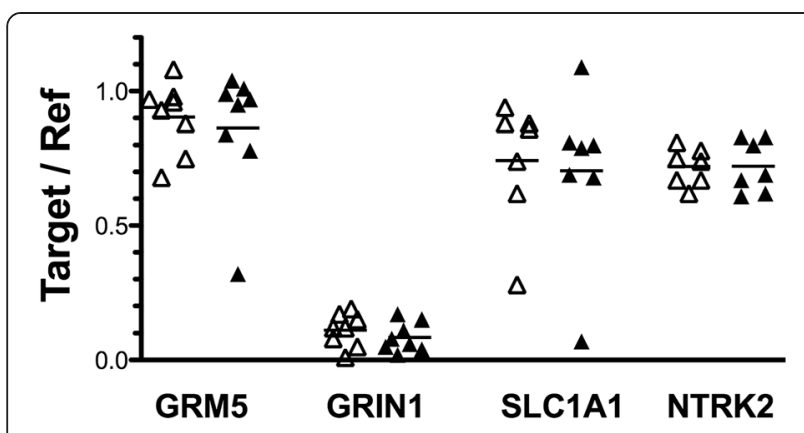

Figure $\mathbf{5}$ Levels of expression of GRM5, GRIN1, SLC1A1, and NTRK2 in pyramidal neurons of BA10. Gene expression was measured in laser captured BA10 pyramidal neurons from typically developing control donors (open symbols) and ASD donors (closed symbols). Gene expression levels are normalized to the averaged levels of expression of references genes (GAPDH and RNA1851). Mean values are noted by horizontal lines. No statistically significant differences were observed.

quality values. These factors were considered extremely important for matching since each would be expected to impact measurements of gene expression levels. In addition, as noted in Methods, tissues from matched pairs of donors were processed on the same day so that RNA storage times were matched. Later, reverse transcription of RNA was performed on the same day for matched donor pairs, and PCR amplifications were performed simultaneously and run on the same plate. Given the meticulous care in the pairing of tissue donors and experiments that were performed on these tissues, we feel most confident with the results of the paired analyses of the resulting data. Alternative unpaired analyses could be used with the argument that all samples are taken from two separate groups of unrelated donors. To consider this, we subjected gene expression data from

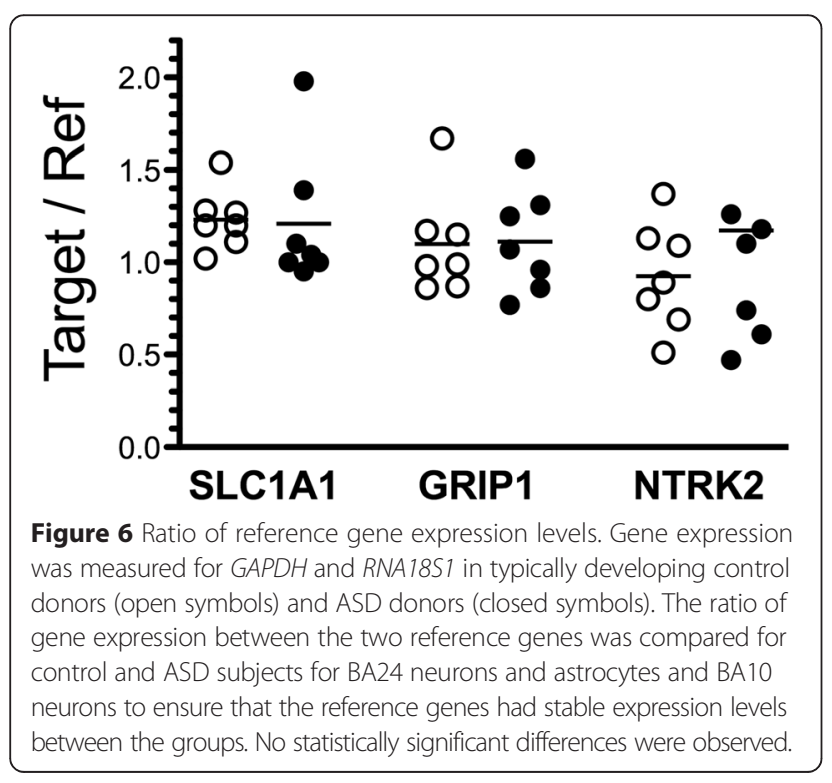

BA24 pyramidal neurons to MANOVA. The results of the MANOVA demonstrated a significant group effect $\left(F=563.3_{(7,1)} ; P=0.03\right)$, with significantly lower expression levels of NTRK2 $(P=0.002)$ in the ASD group as compared to the control group, similar to that observed in the paired analysis above. In addition, expression levels of GRIN1 $(P=0.009)$ and GRM8 $(P=0.02)$ were lower in ASD as compared to control donors.

\section{Discussion}

This study examined the expression of genes involved in glutamatergic neurotransmission in ASD. We found a robust reduction in expression of a neurotrophin receptor gene $(N T R K 2)$ in pyramidal neurons dissected by LCM from ACC tissue of donors with ASD when compared to typically developing controls. In addition, there were trends towards low levels of expression of genes encoding a metabotropic glutamate receptor (GRM8), an ionotropic glutamate receptor subunit (GRIN1), a glutamate transporter (SLC1A1), and a glutamate receptor anchoring protein (GRIP1). This is the first study to use LCM to isolate a specific cell population to examine the molecular pathology of ASD. The use of LCM to capture distinct cell populations provided cellular resolution to the assessment of pathology and permitted identification of gene expression changes that we were unable to detect using homogenates of the same brain region in the same subjects. The difference in the results of LCM of single cell populations versus homogenates of tissues is likely the result of overlapping gene expression in other unaffected cell types that in essence dilute cell-specific gene expression changes, as has been demonstrated in research on Alzheimer's disease $[43,44]$. The gene expression deficits identified in the present study were restricted to ACC pyramidal neurons and not found in surrounding astrocytes in the same brain region, nor in pyramidal neurons in BA10 of the prefrontal cortex, suggesting the presence of a cell- and region-specific pathology in ASD.

A striking finding of this study was the decreased expression of the growth factor receptor gene NTRK2 in ACC pyramidal neurons from ASD donors. This gene encodes the high-affinity tyrosine kinase $B$ receptor, TrkB, the primary ligand for which is BDNF, but neurotrophin-3 (NT-3) and neurotrophin-4/5 (NT-4/5) also can signal through the TrkB receptor $[45,46]$. TrkB signaling is critical to cell function, with ligand-activated TrkB engaging several intracellular signaling pathways including mitogenactivated protein kinase (MAPK), phosphoinositide 3kinase (PI3), and extracellular-signal-regulated kinase (ERK) that are important in neurotransmission, plasticity, and differentiation [47]. Other evidence of abnormal BDNF/TrkB signaling in ASD includes several reports of elevated peripheral and central BDNF levels in ASD [34,48-51]. Given the multitude of effectors of 
TrkB receptor signaling, reduced TrkB signaling in ASD could have severe consequences on brain development and function. NTRK2-deficient mice die soon after birth [52,53], but mice with a conditional loss of NTRK2 expression exhibit a wide variation in behavior ranging from social deficits to antisocial behavior (as reviewed by Lindholm and Castrén, 2014 [54]). It is noteworthy that the $\mathrm{BTBR} \mathrm{T}^{+} t f / J$ mouse model for ASD exhibits reduced TrkB protein expression [55]. Defective TrkB signaling has been shown in Angelman [56] and Fragile X [57] mouse models, both of which are syndromes with a high incidence of autistic features. The present study provides further support for a role of deficient BDNF/TrkB signaling in ASD, specifically implicating reduced TrkB signaling in ACC pyramidal neurons.

A genetic association between polymorphisms of NTRK2 and ASD has been identified [34]. The most strongly associated polymorphisms in ASD were found in the intronspanning regions of NTRK2 [30]. It seems unlikely that a polymorphism in NTRK2 would account for the reduction in NTRK2 expression in pyramidal cells observed in the present study since one would expect the polymorphic NTRK2 to similarly affect NTRK2 expression in astrocytes and pyramidal neurons in other brain regions. Of the cells studied, we found the reduction of NTRK2 expression only in ACC pyramidal neurons.

Cells used for this study were dissected from neocortical layer III of the ACC where both changes in cell size and packing density have been demonstrated previously [21]. An elevated number of neurons detected in layer VI of the ASD ACC have been interpreted as a possible incomplete migration of neurons during development [21]. Mini-column disturbances, that is, reduced column width and increased column number in ASD, have been shown in the ACC with pyramidal neurons demonstrating misalignment [58]. Stoner et al. [59] recently reported abnormal columnar architecture in several cortical regions involving all six cortical layers, based on abnormal gene expressions and areas referred to as "patches" [59] with low gene expression levels. The ACC was not investigated in this later study. In addition, an increase in spine density of pyramidal neurons in frontal, parietal, and temporal lobes has been reported for ASD [60], although spine density specifically in the ACC has not been studied to date. Interestingly, BDNF/TrkB signaling plays a prominent role in synaptogenesis and synaptic remodeling of excitatory neurons during development $[61,62]$. Hence, low levels of NTRK2 expression in glutamatergic pyramidal neurons in ASD observed in the present study may be etiologically linked to abnormalities in cortical columnar architecture in ASD previously reported.

Abnormalities in neurotransmitter signaling networks are likely to be a core component of the cellular neurobiology of ASD. Gene expression changes in synapse- related genes have been demonstrated in disorders that have autistic features such as Angelman syndrome and Fragile X syndrome (as reviewed by Ebert and Greenberg, 2013 [63]). We chose to interrogate potential expression changes in several genes associated with excitatory amino acid cell signaling in autism. Four of these genes, SLC1A1, GRIP1, GRIN1, and GRM8, demonstrated trends towards reduced expression in ACC pyramidal neurons in ASD. SLC1A1 is responsible for glutamate uptake from the synapse by pyramidal neurons while GRIP1 anchors AMPA receptors to the cell membrane. GRIN1 is the mandatory subunit of the N-methyl-D-aspartate (NMDA) receptor and GRM8 inhibits the adenylyl cyclase/cAMP pathway and decreases the likelihood of cell death associated with excess NMDA signaling [64]. The decreased expression of GRM8 receptor in ASD, if accompanied by reduced GRM8 receptor, could leave pyramidal cells susceptible to neurotoxic effects of elevated glutamate signaling through the NMDA receptor. This neurotoxic effect could be amplified further if reduced SLC1A1 expression observed in ASD results in less glutamate transporter activity at the glutamatergic synapse, thereby further increasing glutamate activation of NMDA receptors on these pyramidal neurons. Reduced GRIP1 expression, as we observed in ASD, could be a compensatory mechanism to override excess NMDA receptor activation by glutamate. Whatever the exact sequence or consequence, abnormal expression levels of these genes imply that excitatory output from the ACC via pyramidal neurons is disrupted in ASD. Because multiple cell types express GRIP1 and NTRK2, it cannot be dismissed that these glutamatergic-related gene expression decreases are only found in excitatory cells. While we did not find GRIP1 or excitatory transporter changes (SLC1A2 and SLC1A3) in the glia cells studied herein, altered expression of these genes could occur in inhibitory neurons of the ACC, which were not studied. Nevertheless, any disruption of glutamate transmission and function implicates a compromise in the delicate balance of inhibitory and excitatory neuronal activity of the ACC. Future studies need to include a more detailed examination of gene expression in other cell types of the ACC.

\section{Limitations}

There were several limitations in the current study that should be taken into consideration. First, like many studies of ASD relying on postmortem brain tissue, the number of donors available was limited and reduced the power of the study. It should be noted that all donors that had a history of seizure disorder were excluded from the study to reduce experimental variability. It is worth noting that our laboratory has observed that the study of gene expression in distinct cell populations captured by LCM results in reduced variability of data, permitting the use of smaller sample sizes to obtain statistical 
significance. ASD is a highly heterogeneous disorder, and there were not enough brain donors to permit us to examine the gene expressions in subgroups of donors based on clinical presentation. Changes in gene expression do not always correlate with changes in the expression of the cognate protein, and often a change in gene expression is not temporally correlated with changes in the cognate proteins. Hence, whether reduced NTRK2 expression in ASD pyramidal neurons results in lower TrkB protein in these cells is not currently known. Nevertheless, altered levels of NTRK2 expression in ASD is highly suggestive of perturbed TrkB signaling in ASD. ASD donors used in the present study were exposed to psychotherapeutic drugs that could alter gene expression, particularly SSRI and risperidol, an atypical antipsychotic. But, repeated administration of the antidepressants tranylcypromine, sertraline, or desipramine to rats increases NTRK2 expression [65]. Hence, reduced NTRK2 expression in the present study is unlikely to reflect previous antidepressant drug therapy. Repeated treatment of rats with risperidol produced no change in TrkB immunoreactivity in the brain [66], again suggesting that psychotherapeutic drug exposure is not a likely mediator of reduced NTRK2 expression in ASD in the present study. Findings herein demonstrate no obvious effect of psychotherapeutic drug exposure on NTRK2 (or GRIN1, GRM8, GRIP1, SLC1A1) expression. Finally, tissue-related physical factors were considered as possible sources of variance in these experiments. However, there were no significant differences between ASD and control donor samples when comparing age, RNA quality (RIN), and postmortem intervals (Table 1 ).

\section{Conclusions}

Multiple gene abnormalities are significantly associated with ASD [67]. It therefore seems likely that multiple etiologies exist that can result in ASD. Brain pathology is downstream to gene polymorphisms and etiologies and more proximal to the pathophysiology underlying the abnormal behaviors. Hence, the neuropathology of ASD may be more limited in terms of variability than the genotypes that can contribute to it, that is, different etiologies may result in a similar structural/neurochemical abnormalities that disrupt function to produce a specific set of behavioral abnormalities. The present study was designed to examine possible disruption of a key neuronal unit, the glutamatergic pyramidal neuron, of the ACC in ASD. The data presented here reveals strong evidence of disrupted neurochemistry of ACC pyramidal neurons, specifically involving glutamatergic neurotransmission and neurotrophic factor (BDNF) signaling through the TrkB receptor. It is tempting to speculate that abnormalities in ACC as revealed in imaging studies of ASD patients [4-7,9,11-16,68] and pathology research using postmortem brain tissues [21-25] are at least partially the result of deficits in pyramidal neuronal function in the ACC as revealed in the present study. Pyramidal neurons in layer III of the ACC synapse with other cortical neurons including inhibitory neurons and long-range pyramidal motor neurons in layer $\mathrm{V}$ and the present findings suggest that neural communication with these other brain areas or pathways is altered in ASD. Interestingly, pyramidal neurons take years to reach maturity, possibly well into adolescence [69], indicating that normal neural development of this cell type may be especially vulnerable to deleterious environmental influences occurring early in life.

\section{Additional files}

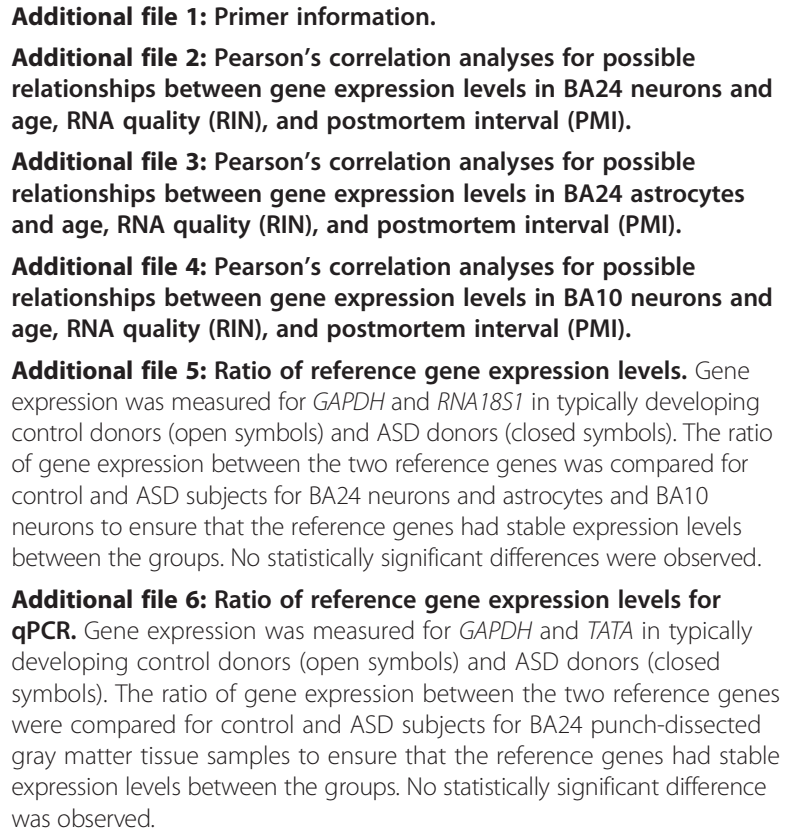

Additional file 3: Pearson's correlation analyses for possible relationships between gene expression levels in BA24 astrocytes and age, RNA quality (RIN), and postmortem interval (PMI).

Additional file 4: Pearson's correlation analyses for possible relationships between gene expression levels in BA10 neurons and age, RNA quality (RIN), and postmortem interval (PMI).

Additional file 5: Ratio of reference gene expression levels. Gene expression was measured for GAPDH and RNA18S1 in typically developing control donors (open symbols) and ASD donors (closed symbols). The ratio of gene expression between the two reference genes was compared for control and ASD subjects for BA24 neurons and astrocytes and BA10 neurons to ensure that the reference genes had stable expression levels between the groups. No statistically significant differences were observed.

Additional file 6: Ratio of reference gene expression levels for qPCR. Gene expression was measured for GAPDH and TATA in typically developing control donors (open symbols) and ASD donors (closed symbols). The ratio of gene expression between the two reference genes were compared for control and ASD subjects for BA24 punch-dissected gray matter tissue samples to ensure that the reference genes had stable expression levels between the groups. No statistically significant difference was observed.

\section{Abbreviations}

ACC: anterior cingulate cortex; ASD: autism spectrum disorder; BA10: Brodmann area 10; BA24: Brodmann area 24; Ct: cycle threshold; EEG: electroencephalogram; LCM: laser capture microdissection; MANOVA: multivariate analysis of variance; PET: positron emission tomography; PMI: postmortem interval; qPCR: real-time quantitative polymerase chain reaction; RIN: RNA integrity number; SPECT: single-photon emission computerized tomography; SSRI: selective serotonin retake inhibitors.

\section{Competing interests}

The authors declare that they have no competing interests.

\section{Authors' contributions}

MJC made significant contributions to experimental work and data analysis. She is responsible for drafting the original manuscript. JDC is responsible for all tissue preparation, immunohistochemistry, and all real-time experimentation of cells used. She is also responsible for the final preparation of the written manuscript. AS managed all experimentation involving gene expression, both endpoint PCR and real-time PCR. KS managed all tissue preparation, immunohistochemistry, and all aspects of laser capture microdissection. GAO was the PI of the work presented. He was responsible for overseeing 
all aspects of the project, including data analysis and manuscript preparation. All authors have read and approved this manuscript.

\section{Acknowledgements}

The authors are grateful to the families who made the choice to consent to donate brain tissue. This invaluable contribution will support many years of ASD research. Human brain tissue was obtained from the Autism Speaks Autism Tissue Program, the Harvard Brain Tissue Resource, and the NICHD Brain and Tissue Bank for Developmental Disorders at the University of Maryland, Baltimore, MD. Written informed consent was obtained from the subject's next of kin for publication of their individual details in this manuscript. The consent forms are held by the above mentioned brain banks and are available for review by the Editor-in-Chief. Autism Speaks pilot grant \#7330 and the National Institutes of Health RR030651 supported this research.

\section{Author details}

${ }^{1}$ Department of Health Sciences, College of Public Health, East Tennessee State University, P.O. Box 70673, Johnson City, TN 37614, USA. ²Department of Biomedical Sciences, James H. Quillen College of Medicine, East Tennessee State University, P.O. Box 70582, Johnson City, TN 37614, USA.

\section{Received: 19 December 2014 Accepted: 24 April 2015 Published online: 16 May 2015}

\section{References}

1. American Psychiatric Association. Diagnostic and statistical manual of mental disorders (DSM V), Fifth. 2013.

2. Jeste SS, Geschwind DH. Disentangling the heterogeneity of autism spectrum disorder through genetic findings. Nat Rev Neurol. 2014;10:74-81.

3. Bush G, Luu P, Posner M. Cognitive and emotional influences in anterior cingulate cortex. Trends Cogn Sci. 2000;4:215-22.

4. Kennedy DP, Courchesne E. Functional abnormalities of the default network during self- and other-reflection in autism. Soc Cogn Affect Neurosci. 2008:3:177-90.

5. Gomot M, Bernard FA, Davis MH, Belmonte MK, Ashwin C, Bullmore ET, et al. Change detection in children with autism: an auditory event-related fMRI study. Neuroimage. 2006;29:475-84.

6. Kana RK, Keller TA, Minshew NJ, Just MA. Inhibitory control in high-functioning autism: decreased activation and underconnectivity in inhibition networks. Biol Psychiatry. 2007;62:198-206.

7. Agam Y, Joseph RM, Barton JJS, Manoach DS. Reduced cognitive control of response inhibition by the anterior cingulate cortex in autism spectrum disorders. Neuroimage. 2010;52:336-47.

8. Fan J, Bernardi S, Van Dam NT, Anagnostou E, Gu X, Martin L, et al. Functional deficits of the attentional networks in autism. Brain Behav. 2012;2:647-60.

9. Dichter GS, Felder JN, Bodfish JW. Autism is characterized by dorsal anterior cingulate hyperactivation during social target detection. Soc Cogn Affect Neurosci. 2009;4:215-26.

10. Sasaki M, Nakagawa E, Sugai K, Shimizu Y, Hattori A, Nonoda Y, et al. Brain perfusion SPECT and EEG findings in children with autism spectrum disorders and medically intractable epilepsy. Brain Dev. 2010;32:776-82.

11. Vlamings PHJM, Jonkman LM, Hoeksma MR, van Engeland H, Kemner C. Reduced error monitoring in children with autism spectrum disorder: an ERP study. Eur J Neurosci. 2008;28:399-406.

12. Sokhadze E, Baruth J, Tasman A, Mansoor M, Ramaswamy R, Sears L, et al. Low-frequency repetitive transcranial magnetic stimulation (rTMS) affects event-related potential measures of novelty processing in autism. Appl Psychophysiol Biofeedback. 2010;35:147-61

13. Santesso DL, Drmic IE, Jetha MK, Bryson SE, Goldberg JO, Hall GB, et al. An event-related source localization study of response monitoring and social impairments in autism spectrum disorder. In: Psychophysiology. 2010.

14. Henderson H, Schwartz C, Mundy P, Burnette C, Sutton S, Zahka N, et al. Response monitoring, the error-related negativity, and differences in social behavior in autism. Brain Cogn. 2006;61:96-109.

15. Nakamura K, Sekine Y, Ouchi Y, Tsujii M, Yoshikawa E, Futatsubashi M, et al. Brain serotonin and dopamine transporter bindings in adults with high-functioning autism. Arch Gen Psychiatry. 2010;67:59-68.

16. Hall GBC, Szechtman H, Nahmias C. Enhanced salience and emotion recognition in Autism: a PET study. Am J Psychiatry. 2003;160:1439-41.
17. Haznedar MM, Buchsbaum MS, Wei TC, Hof PR, Cartwright C, Bienstock CA, et al. Limbic circuitry in patients with autism spectrum disorders studied with positron emission tomography and magnetic resonance imaging. Am J Psychiatry. 2000;157:1994-2001.

18. Haznedar MM, Buchsbaum MS, Metzger M, Solimando A, Spiegel-Cohen J, Hollander E. Anterior cingulate gyrus volume and glucose metabolism in autistic disorder. Am J Psychiatry. 1997;154:1047-50.

19. Thakkar KN, Polli FE, Joseph RM, Tuch DS, Hadjikhani N, Barton JJS, et al. Response monitoring, repetitive behaviour and anterior cingulate abnormalities in autism spectrum disorders (ASD). Brain. 2008;131(Pt 9):2464-78.

20. Barnea-Goraly N, Kwon H, Menon V, Eliez S, Lotspeich L, Reiss AL. White matter structure in autism: preliminary evidence from diffusion tensor imaging. Biol Psychiatry. 2004;55:323-6.

21. Simms ML, Kemper TL, Timbie CM, Bauman ML, Blatt GJ. The anterior cingulate cortex in autism: heterogeneity of qualitative and quantitative cytoarchitectonic features suggests possible subgroups. Acta Neuropathol. 2009;118:673-84.

22. Suda S, Iwata K, Shimmura C, Kameno Y, Anitha A, Thanseem I, et al. Decreased expression of axon-guidance receptors in the anterior cingulate cortex in autism. Mol Autism. 2011:2:14

23. Thanseem I, Nakamura K, Miyachi T, Toyota T, Yamada S, Tsujii M, et al. Further evidence for the role of MET in autism susceptibility. Neurosci Res. 2010;68:137-41.

24. Oblak A, Gibbs T, Blatt G. Decreased GABAA receptors and benzodiazepine binding sites in the anterior cingulate cortex in autism. Autism Res. 2009;2:205-19.

25. Oblak A, Gibbs T, Blatt G. Decreased GABA(B) receptors in the cingulate cortex and fusiform gyrus in autism. J Neurochem. 2010;114:1414-23.

26. Thanseem I, Anitha A, Nakamura K, Suda S, Iwata K, Matsuzaki H, et al. Elevated transcription factor specificity protein 1 in autistic brains alters the expression of autism candidate genes. Biol Psychiatry. 2012;71:410-8.

27. Dölen G, Osterweil E, Rao BSS, Smith GB, Auerbach BD, Chattarji S, et al. Correction of fragile $X$ syndrome in mice. Neuron. 2007:56:955-62.

28. Purcell $A E$, Jeon $\mathrm{OH}$, Zimmermana W, Blue ME, Pevsner J. Postmortem brain abnormalities of the glutamate neurotransmitter system in autism. Neurology. 2001;57:1618-28.

29. Barnby G, Abbott A, Sykes N, Morris A, Weeks DE, Mott R, et al. Candidate-gene screening and association analysis at the autism-susceptibility locus on chromosome 16p: evidence of association at GRIN2A and ABAT. Am J Hum Genet. 2005;76:950-66.

30. Rainey L, van der Walt JH. The anaesthetic management of autistic children. Anaesth Intensive Care. 1998;26:682-6.

31. Pinto D, Pagnamenta AT, Klei L, Anney R, Merico D, Regan R, et al. Functional impact of global rare copy number variation in autism spectrum disorders. Nature. 2010;466:368-72.

32. Jamain S, Betancur C, Quach H, Philippe A, Fellous M, Giros B, et al. Linkage and association of the glutamate receptor 6 gene with autism. Mol Psychiatry. 2002;7:302-10.

33. Browne CA, Lucki I. Antidepressant effects of ketamine: mechanisms underlying fast-acting novel antidepressants. Front Pharmacol. 2013:4:161.

34. Correia CT, Coutinho aM, Sequeira aF, Sousa IG, Lourenço Venda L, Almeida JP, et al. Increased BDNF levels and NTRK2 gene association suggest a disruption of BDNF/TrkB signaling in autism. Genes Brain Behav. 2010;9:841-8.

35. Auer H, Lyianarachchi S, Newsom D, Klisovic MIM, Marcucci G, Marcucci U, et al. Chipping away at the chip bias : RNA degradation in microarray analysis. Nat Genet. 2003:35:292-3.

36. Ordway GA, Szebeni A, Duffourc MM, Dessus-Babus S, Szebeni K. Gene expression analyses of neurons, astrocytes, and oligodendrocytes isolated by laser capture microdissection from human brain: detrimental effects of laboratory humidity. J Neurosci Res. 2009;87:2430-8.

37. Chandley MJ, Szebeni K, Szebeni A, Crawford J, Stockmeier CA, Turecki G, et al. Gene expression deficits in pontine locus coeruleus astrocytes in men with major depressive disorder. J Psychiatry Neurosci. 2013;38:120110.

38. Zuker M. Mfold web server for nucleic acid folding and hybridization prediction. Nucleic Acids Res. 2003;31:3406-15.

39. Xiang L, Szebeni K, Szebeni A, Klimek V, Stockmeier CA, Karolewicz B, et al. Dopamine receptor gene expression in human amygdaloid nuclei: elevated D4 receptor mRNA in major depression. Brain Res. 2008;1207:214-24.

40. Livak KJ, Schmittgen TD. Analysis of relative gene expression data using real-time quantitative PCR and the 2(-Delta Delta C(T)) Method. Methods. $2001 ; 25: 402-8$ 
41. Holm S. A simple sequentially rejective multiple test procedure. Scand J Stat. 1979:6:65-70.

42. Holm-Bonferroni sequential correction: an EXCEL calculator [http:// www.researchgate.net/publication/236969037_Holm-Bonferroni_Sequential_ Correction_An_EXCEL_Calculator]

43. Ginsberg SD, Elarova I, Ruben M, Tan F, Counts SE, Eberwine JH, et al. Single-cell gene expression analysis: implications for neurodegenerative and neuropsychiatric disorders. Neurochem Res. 2004;29:1053-64.

44. Ginsberg SD, Che S. Expression profile analysis within the human hippocampus: comparison of CA1 and CA3 pyramidal neurons. J Comp Neurol. 2005:487:107-18

45. Klein R, Nanduri V, Jing SA, Lamballe F, Tapley P, Bryant S, et al. The trkB tyrosine protein kinase is a receptor for brain-derived neurotrophic factor and neurotrophin-3. Cell. 1991;66:395-403.

46. Klein R, Lamballe F, Bryant S, Barbacid M. The trkB tyrosine protein kinase is a receptor for neurotrophin-4. Neuron. 1992;8:947-56.

47. Boulle F, Kenis G, Cazorla M, Hamon M, Steinbusch HWM, Lanfumey L, et al. TrkB inhibition as a therapeutic target for CNS-related disorders. Prog Neurobiol. 2012;98:197-206.

48. Connolly AM, Chez M, Streif EM, Keeling RM, Golumbek PT, Kwon JM, et al. Brain-derived neurotrophic factor and autoantibodies to neural antigens in sera of children with autistic spectrum disorders, Landau-Kleffner syndrome, and epilepsy. Biol Psychiatry. 2006;59:354-63.

49. Miyazaki K, Narita N, Sakuta R, Miyahara T, Naruse H, Okado N, et al. Serum neurotrophin concentrations in autism and mental retardation: a pilot study. Brain Dev. 2004;26:292-5.

50. Nelson PG, Kuddo T, Song EY, Dambrosia JM, Kohler S, Satyanarayana G, et al. Selected neurotrophins, neuropeptides, and cytokines: developmental trajectory and concentrations in neonatal blood of children with autism or Down syndrome. Int J Dev Neurosci. 2006;24:73-80.

51. Perry EK, Lee ML, Martin-Ruiz CM, Court JA, Volsen SG, Merrit J, et al. Cholinergic activity in autism: abnormalities in the cerebral cortex and basal forebrain. Am J Psychiatry. 2001;158:1058-66.

52. Klein R, Smeyne RJ, Wurst W, Long LK, Auerbach BA, Joyner AL, et al. Targeted disruption of the trkB neurotrophin receptor gene results in nervous system lesions and neonatal death. Cell. 1993;75:113-22.

53. Rohrer B, Blanco R, Marc RE, Lloyd MB, Bok D, Schneeweis DM, et al. Functionally intact glutamate-mediated signaling in bipolar cells of the TRKB knockout mouse retina. Vis Neurosci. 2004;21:703-13.

54. Lindholm JSO, Castrén E. Mice with altered BDNF signaling as models for mood disorders and antidepressant effects. Front Behav Neurosci. 2014;8:143.

55. Scattoni ML, Martire A, Cartocci G, Ferrante A, Ricceri L. Reduced social interaction, behavioural flexibility and BDNF signalling in the BTBR T+tf/J strain, a mouse model of autism. Behav Brain Res. 2013;251:35-40.

56. Cao C, Rioult-Pedotti MS, Migani P, Yu CJ, Tiwari R, Parang K, et al. Impairment of TrkB-PSD-95 signaling in Angelman syndrome. PLoS Biol. 2013;11, e1001478.

57. Uutela M, Lindholm J, Rantamäki T, Umemori J, Hunter K, Võikar V, et al. Distinctive behavioral and cellular responses to fluoxetine in the mouse model for Fragile X syndrome. Front Cell Neurosci. 2014;8:150.

58. Casanova MF, Buxhoeveden DP, Brown C. Clinical and macroscopic correlates of minicolumnar pathology in autism. J Child Neurol. 2002;17:692-5.

59. Stoner R, Chow ML, Boyle MP, Sunkin SM, Mouton PR, Roy S, et al. Patches of disorganization in the neocortex of children with autism. N Engl J Med. 2014;370:1209-19.

60. Avino TA, Hutsler JJ. Abnormal cell patterning at the cortical gray-white matter boundary in autism spectrum disorders. Brain Res. 2010;1360:138-46.

61. Ohira K, Hayashi M. A new aspect of the TrkB signaling pathway in neural plasticity. Curr Neuropharmacol. 2009;7:276-85.

62. Luikart BW, Parada LF. Receptor tyrosine kinase B-mediated excitatory synaptogenesis. Prog Brain Res. 2006;157:15-24.

63. Ebert D, Greenberg M. Activity-dependent neuronal signalling and autism spectrum disorder. Nature. 2013;493:327-37.

64. Ambrosini A, Bresciani L, Fracchia S, Brunello N, Racagni G. Metabotropic glutamate receptors negatively coupled to adenylate cyclase inhibit N-methylD-aspartate receptor activity and prevent neurotoxicity in mesencephalic neurons in vitro. Mol Pharmacol. 1995;47:1057-64.

65. Nibuya M, Morinobu S, Duman RS. Regulation of BDNF and trkB mRNA in rat brain by chronic electroconvulsive seizure and antidepressant drug treatments. J Neurosci. 1995;15:7539-47.
66. Angelucci F, Mathé AA, Aloe L. Brain-derived neurotrophic factor and tyrosine kinase receptor TrkB in rat brain are significantly altered after haloperidol and risperidone administration. J Neurosci Res. 2000;60:783-94.

67. Betancur C. Etiological heterogeneity in autism spectrum disorders: more than 100 genetic and genomic disorders and still counting. Brain Res. 2011;1380:42-77.

68. Palmen SJMC, van Engeland H, Hof PR, Schmitz C. Neuropathological findings in autism. Brain. 2004;127(Pt 12):2572-83.

69. Huttenlocher PR. Myelination and the development of function in immature pyramidal tract. Exp Neurol. 1970;29:405-15.

\section{Submit your next manuscript to BioMed Central and take full advantage of:}

- Convenient online submission

- Thorough peer review

- No space constraints or color figure charges

- Immediate publication on acceptance

- Inclusion in PubMed, CAS, Scopus and Google Scholar

- Research which is freely available for redistribution

Submit your manuscript at www.biomedcentral.com/submit
C BioMed Central 